\title{
As dimensões do cuidado pré-natal na consulta de enfermagem*
}

\author{
The dimensions of prenatal care embodied in nursing consultation \\ Las dimensiones del cuidado prenatal en la consulta de enfermería
}

\section{Helena Eri Shimizu', Maria Goreti de Lima'}

'Universidade de Brasília. Faculdade de Ciências da Saúde. Programa de Pós-Graduação em Ciências da Saúde. Brasília, DF

Submissão: $07 / 08 / 2008$

Aprovação: 03/05/2009

\section{RESUMO}

O estudo teve como objetivos analisar as representações sociais das gestantes acerca da gestação e a atenção recebida na consulta de enfermagem do pré-natal. Foram realizadas entrevistas semi-estruturadas com Quinze gestantes, Que foram analisados com o auxílio do software ALCEST. Identificaram-se três eixos temáticos: a vivência da gravidez, constituída pelas classes: o impacto e as mudanças provocadas pela gravidez, as orientações recebidas sobre a gravidez com as classes percepção da consulta de enfermagem e do planejamento familiar e os cuidados com o bebê com as classes como cuidar do recém nascido e como garantir uma boa amamentação. A consulta de enfermagem abarca as dimensões psicossociais dos cuidados com a gestante e com recém-nascido.

Descritores: Pré-natal; Gravidez; Enfermagem, Atenção primária à saúde, Psicologia social

\begin{abstract}
This study aimed at analyzing the social representation of pregnant women about pregnancy, regarding care provided in nursing consultations during the prenatal period. A Qualitative study conducted by semi-structured interviews, with fifteen pregnant women at the nursing consultations of the prenatal program regarding the care provided, analyzed by the ALCEST software. Three thematic axes were identified: the experience of pregnancy, constituted by the classes: the impact and the changes due to pregnancy; the orientations provided about pregnancy, with the classes: perception of the nursing consultation and family planning; and the approaches baby care, with the classes: how to take care of the new-born and how to assure good breastfeeding. A nursing consultation covers different dimensions psychosocial to pregnancy and to the newborn.
\end{abstract}

Descriptors: Prenatal care; Pregnancy; Nursing, Primary health care, Social psychology.

\section{RESUMEN}

Esto estudio objectivó analizar las representaciones sociales de las gestantes acerca de la atención recibida en la consulta de enfermería del prenatal. Estudio cualitativo de la atención recibida, realizado por medio de entrevistas semi-estructuradas y analizadas con auxilio del software ALCEST, con Quince gestantes atendidas en la consulta de enfermería del programa de prenatal. Se identificaron tres ejes temáticos: la vivencia del embarazo, constituida por las clases: el impacto y los cambios provocados por el embarazo; las orientaciones recibidas sobre el embarazo, con las clases: percepción de la consulta de enfermería y del planeamiento familiar; y el los cuidados del bebé, con las clases: cómo cuidar al recién nacido y cómo garantizar un buen amamantamiento. La consulta de enfermería abarca diversas dimensiones psicosocial tanto de los cuidados durante la gestación como con el recién nacido.

Descriptores: Atención prenatal; Embrarazo; Enfermería, Atención primaria de salud, Psicología social.

* Artigo abstraído da dissertação de mestrado intitulada: Representações sociais das gestantes sobre a gravidez e a consulta de enfermagem no pré-natal, apresentada ao Programa de Ciências da Saúde da Universidade de Brasília, em 2006. 


\section{INTRODUÇÃO}

Na história da Saúde Pública, a atenção materno-infantil tem sido considerada uma área prioritária, principalmente no Que diz respeito aos cuidados da mulher durante a gestação, Que engloba: o pré-natal, o parto e o puerpério, a fim de manter um ciclo gravídico-puerperal com o menor risco possível para o binômio mãe-filho.

Dentre elas, destaca-se o Programa Nacional de Atenção Integral à Saúde da Mulher (PAISM) Que propôs uma nova e diferenciada abordagem, com ênfase no atendimento à saúde reprodutiva das mulheres no âmbito da atenção integral, com vistas ao aperfeiçoamento do controle do pré-natal, parto e puerpério ${ }^{(1)}$.

No Que diz respeito à atenção do pré-natal, o MS, por meio do PAISM, estabeleceu os seguintes procedimentos para: captar a gestante na comunidade, fazer os controles periódicos, contínuos; garantir as consultas, bem como reuniões educativas, prover área física adequada, equipamento e instrumental mínimo; oferecer medicamentos básicos e apoio laboratorial ${ }^{(2)}$.

Embora estudos demonstrem os benefícios do acompanhamento pré-natal sobre a saúde da gestante e do recém-nascido, Que contribuem para a redução da mortalidade materna, baixo peso ao nascer e mortalidade peri-natal ${ }^{(3,4)}$, a cobertura da consulta prénatal, especificamente o número de consulta é deficiente, e verificase desigualdade entre as regiões do país: norte $26,55 \%$, Nordeste 34,9 , Sudeste $60,54 \%$, Sul $61,05 \%$, Centro Oeste $55,85 \%$, o Que totaliza 49, $14 \%$ no país ${ }^{(5)}$.

Estes dados indicam Que há Que se pensar em formas de expandir o acesso das gestantes aos serviços de saúde, bem como em melhorar a Qualidade das consultas, principalmente fortalecendo o acolhimento, a fim de garantir a adesão ao programa pré-natal.

Observa-se Que no Brasil ainda é muito forte a representação social das gestantes sobre o processo gestacional como um fenômeno natural, Que contribui para a falta de cuidado na gravidez, a não aderência e evasão do programa pré-natal, o Que tem culminado na alta incidência de distúrbios gestacionais graves ${ }^{(6)}$.

A consulta de enfermagem apresenta-se como um instrumento de suma importância, pois têm como finalidade garantir a extensão da cobertura e melhoria da Qualidade pré-natal, principalmente por meio da introdução das ações de preventivas e promocionais as gestantes. É requerido, do profissional além da competência técnica, sensibilidade para compreender o ser humano e o seu modo de vida e habilidade de comunicação, baseada na escuta e na ação dialógica ${ }^{(7)}$

São escassos os estudos sobre a Qualidade da assistência prénatal ${ }^{(3)}$, considerando as gestantes protagonistas do processo gestacional e da avaliação da atenção recebida, a fim de garantir a aderência a essa assistência, em busca da integralidade da atenção.

Este estudo tem como objetivo geral analisar as representações das gestantes acerca da gestação e da atenção recebida na consulta de enfermagem do pré-natal a fim de oferecer subsídios para melhorar a Qualidade da assistência prestada. E como objetivos específicos identificar e analisar as representações sociais das gestantes sobre a gravidez e analisar as contribuições e limitações da Consulta de Enfermagem no Pré-Natal percebidas pelas gestantes para enfrentamento do processo de gestação, parto e puerpério.

\section{METODOLOGIA}

Optou-se pelo desenho com método Qualitativo para compreender as representações sociais das gestantes acerca da gestação, bem como a percepção dos cuidados recebidos na consulta de enfermagem de pré-natal. Entende-se por representações sociais a forma como os grupos sociais interpretam a realidade Que rege as suas relações com o seu meio físico e social e Que orientam as suas condutas e práticas.

Foram realizadas entrevistas com Quinze gestantes, Que obedeceram aos seguintes critérios de inclusão/exclusão: ser maior de dezoito anos e ter realizado no mínimo duas consultas de enfermagem no Centro de Saúde de Brasília noll (CSB-N. II), pertencente à Secretaria de Estadual de Saúde do DF. Utilizou-se um roteiro Que visava estimular a expressão sobre a consulta de enfermagem, os conhecimentos Que absorveram sobre processo gestacional, parto e puerpério, como forma de garantir condições necessárias para sua saúde e a Do recém-nascido. As entrevistas foram gravadas, com a autorização das participantes, e posteriormente transcritas na íntegra.

Para sistematizar os dados, optou-se pelo uso do software ALCESTE (Anályse Lexicale por Contexte d' um Esemble de Segments de Texte). Esse software foi desenvolvido na França por Max Reinert, em 1983. Por essa ferramenta, um corpus é analisado por Unidade de Contexto Elementar - U.C.E, registrada por seQüência de palavras, ou frase, de extensão variável. Dentro de cada uma delas é desenvolvido um procedimento de análise de recorrência de palavras, sendo considerado válido um critério de freqüência mínima. Infere-se Que onde há alta recorrência e valores de $\mathrm{X}^{2}$ significativos, existe núcleo de sentido potencialmente válido ${ }^{(8)}$.

Esses dados permitem reconstituir o "discurso das falas coletivas", utilizando a relação entre as palavras, a freQüência e o percentual com Que aparecem e seu significado, observado a partir dos enunciados Que permitem identificar o contexto em Que se inserem as classes de palavras. Com isso, permite-se o acesso à contextualização do vocabulário mais significativo das classes.

Conforme determinação da Resolução 196/96 do Conselho Nacional de Saúde, o Projeto de pesquisa foi aprovado pelo Comitê de Ética da Secretaria Estadual de Saúde do Distrito Federal (SES/ DF) e todos os participantes assinaram o Termo de Consentimento Livre e Esclarecido.

\section{RESULTADOS E DISCUSSÃO}

Os resultados da análise lexical das Quinze entrevistas mostraram Que as falas das gestantes se organizaram, conforme demonstra o dendograma (figura 1), em torno de três eixos principais, a saber: Vivenciando a gravidez, Recebendo orientações sobre a gestação e Cuidando do bebê.

O dendograma demonstra Que o software ALCEST, a partir do conteúdo total dividiu o material discursivo em dois grandes blocos, posteriormente em três grandes novas divisões e, finalmente, duas últimas. Assim, as classes 1 e 4 , as classes 2 e 6 , e as classes 3 e 5 , possuem significados comuns. Ressalta-se, Que os sentidos e idéias específicas justificam a separação entre as distintas classes.

Do total de UCE Que o programa produziu em todo o corpus textual, 304 foram classificadas e processadas em seis classes temáticas. 


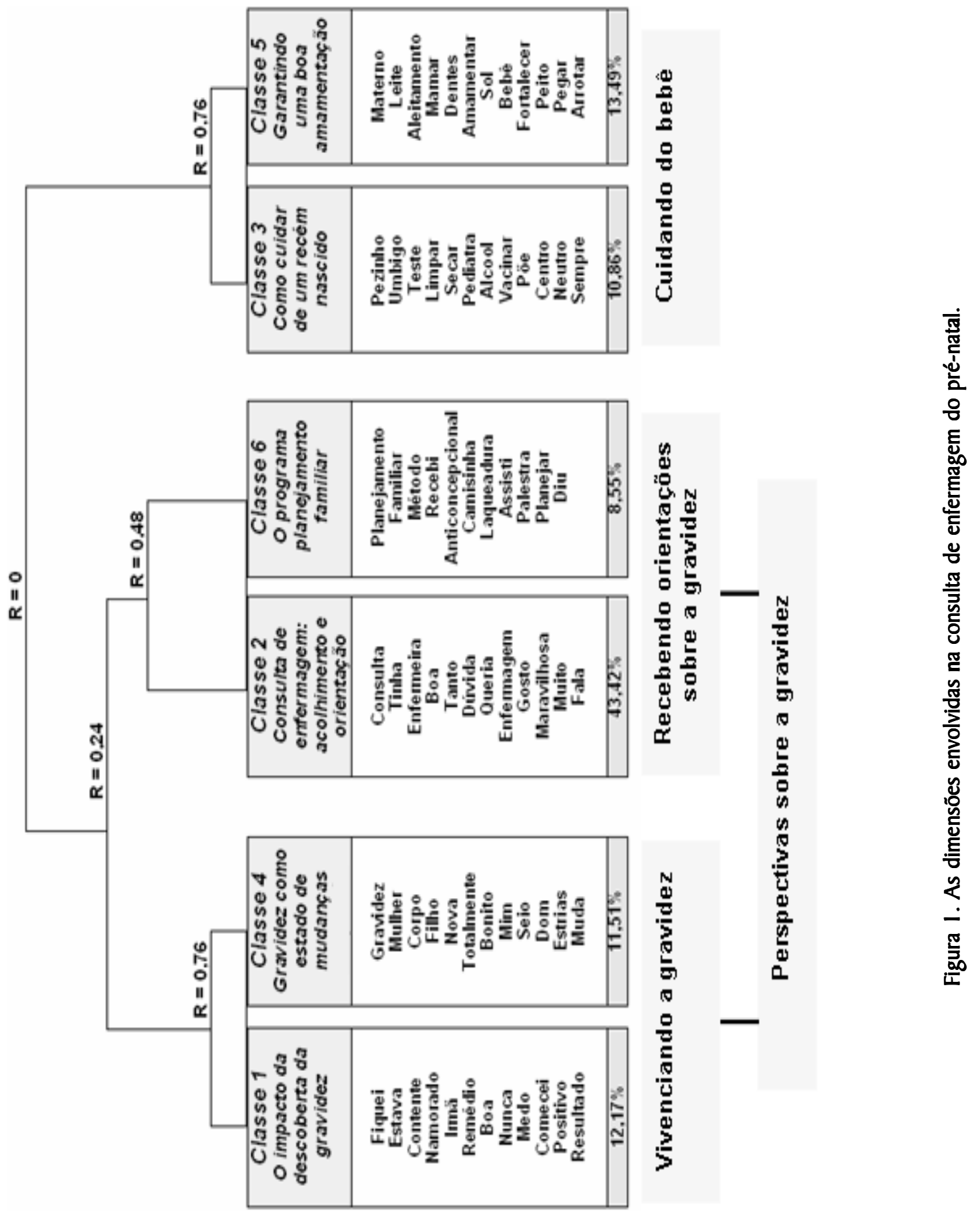


Globalmente, os resultados se mostram homogêneos em relação ao percentual de UCE por classe temática, com exceção da classe 2, Que é representada por 43,42\% e as demais apresentam os seguintes valores ; classe $1: 12,17 \%$, classe $3: 10,86 \%$, classe $4: 11,51 \%$, classe $5: 13,39 \%$ e classe $6: 8,55 \%$.

No primeiro eixo temático: vivenciando a gravidez, composto por duas classes temáticas - classe 1 - o impacto da descoberta da Gravidez e a classe 4- gravidez como estado de mudança - apreende-se os conteúdos lexicais relacionados aos sentimentos suscitados pela descoberta da gravidez, as diversas mudanças percebidas: psicológicas, biológicas e sociais provocadas por essa nova condição.

Verifica-se Que a descoberta da gravidez comumente gera diversos tipos de emoções como: surpresa, alegria e algumas vezes o medo. Fatores como o planejamento pessoal e, principalmente, o desejo da mulher em relação a maternidade contribuem para o predomínio da vivência de sentimentos positivos, mas Quando ocorre o contrário, sobretudo a ausência do apoio do companheiro ou da família dispõe a vivência da insegurança e solidão.

A gravidez é representada como um momento de profundas e complexas mudanças biológicas, fisiológicas e psicológicas. Essas mudanças aparecem, simultaneamente, com significados ora positivos ora negativos no desenvolvimento da gravidez, e Que são de difícil aceitação, favorecendo, de acordo com o pensamento de Barine $^{(9)}$, os componentes de ambivalência e regressão psicológica.

[...] a gravidez vem assim com muita mudança [...] e sem contar das transformações, a gente fica meio chata, fica mais impaciente, apesar da gente está sendo orientada e tudo fica meio perdida, com essa gravidez e com esse filho que vai chegar, e assim a barriga da gente Que cresce, e a gente fica meio em depressão (Sujeito 6).

A percepção de Que um ser está se desenvolvendo em seu ventre possibilita às gestantes vivências de sentimentos de poder; o crescimento dos seios o de aumento da beleza feminina. Nessa perspectiva a gestação é representada como um fenômeno Que vai além da função reprodutiva, comumente associada a algo divino e sublime. A expressão de estar grávida significa dar visibilidade à feminilidade e reafirmar a fecundidade como algo que enaltece a condição da mulher.

[... gravidez pra mim, eu acho que é uma benção de Deus, é um dom divino, é uma maravilha a mulher ficar grávida, gerar uma vida, gerar um filho (Sujeito I0).

Todavia, percebe também que a maioria das mulheres também experimenta o medo da transformação negativa do seu corpo, ou seja, de Que as marcas da gravidez, como as estriais e o excessos de pele, fieuem para o resto de sua vida. Essa experiência é vivida de forma muito angustiante.

Cada mulher vive diferentes sentimentos, de acordo com as suas concepções, percepções e realidade social. Como ressalta Oliveira ${ }^{(1)}$, Mas percebe-se Que a gravidez é representada como um momento delicado na vida de uma mulher, diferentemente do discurso social idealizante, Que apregoa ser este um momento sempre de total felicidade $^{(10)}$, tal idealização da gestação deixa de considerar as dificuldades que muitas mulheres vivenciam nesse momento.
No segundo eixo, recebendo orientações sobre a gravidez, percebem-se duas casses temáticas: Classe 2- Consulta de Enfermagem e Classe 6 - Planejamento Familiar, são tratadas as representações das gestantes sobre a consulta de enfermagem e sobre orientações recebidas acerca do planejamento familiar.

As gestantes demonstraram Que conheceram a consulta de enfermagem durante o pré-natal. Assim sendo, inicialmente, elas tinham a percepção de Que era um procedimento complementar ao trabalho do médico. Contribui para esse tipo de percepção, o fato de grande parte da população ainda desconhecer o trabalho da enfermeira na prevenção de doenças e na promoção da saúde ${ }^{(11)}$.

As gestantes, contudo, a medida Que se submeterem a consulta, passaram avaliá-la positivamente, sobretudo, por permitir a obtenção de informações sobre processo gestacional, Que contribui para o seu auto-conhecimento, identificação dos sinais e sintomas possíveis em cada período, redução de alguns medos Que envolvem o processo gestacional.

[...] com essa consulta da enfermeira a gente acompanha mais a gravidez, qualquer coisa diferente que se veja, ela diz pra observar, que cada mês é diferente do outro, que a cada mês a barriga cresce, o bebê mexe mais, e como sou mãe de primeira viagem acho importante saber o porQuê de tudo (Sujeito I3).

Além disso, a consulta de enfermagem é reconhecida como um espaço de acolhimento porQue possibilita o diálogo, permitindo a livre expressão de dúvidas, de sentimentos, e de experiências, estreitando o vínculo entre a enfermeira e a gestante.

Nesse sentido percebe-se Que a comunicação dialógica representa um pilar na relação enfemeira-gestante, principalmente para favorecer à gestante compreensão desse complexo processo, empoderando-a para enfrentá-lo com mais tranQüilidade.

Segundo as representações das gestantes, as orientações recebidas permitiram ampliar o conhecimento não apenas do processo gestacional, mas também do parto, Que é um momento bastante temido. $\mathrm{O}$ parto, pode ser associado a morte e a outras complicações, necessitando dessa maneira, de um preparo, em que considerem os procedimentos técnicos, os aspectos emocionais e culturais.

Na classe 6 identificam-se os conteúdos absorvidos pelas gestantes acerca do planejamento familiar, Que são trabalhadas em forma de atividade educativa e entendida como contígua a consulta de enfermagem.

Os depoimentos evidenciam Que a temática é tratada de modo informativo, por meio do ensino da adoção de métodos anticoncepcionais para o controle do número de filhos.

[...] o planejamento familiar é pra gente aprender a evitar uma gravidez não desejada, saber Quantos filhos a gente vai Querer ter [...] é como usar os métodos que você vai Querer tá usando, como o DIU, anticoncepcional, camisinha e a laqueadura (Sujeito 3).

Os métodos mais enfatizados são os medicamentosos como a pílula, DIU, injeção de hormônios. Dos métodos naturais destacase a camisinha, mas não se percebeu o ensino sobre conhecimento mais aprofundado sobre o funcionamento do corpo da mulher.

Alem desses métodos, verificou-se Que esterilização feminina 
(laqueadura de trompas), como demonstra também outros estudos ${ }^{(12)}$ foi mencionada pelas mulheres deste estudo como uma opção importante para resolver as dificuldades enfrentadas com outros métodos, sobretudo pelas multíparas.

A laqueadura tubária é o método mais utilizado no Brasil, notadamente nas regiões Centro-Oeste, Norte do Estado do Rio de Janeiro e região Nordeste ${ }^{(13)}$. Destaca-se Que, a Queda da fecundidade aliada à realização da laqueadura sem critérios adequados tem levado ao fato de Que em oito Estados da Federação e no Distrito Federal a média de 2,1 filhos por mulher tem sido menor Que a taxa de reposição, o mínimo necessário para Que a população permaneça estável ${ }^{(14)}$.

Verifica-se Que o planejamento familiar é abordado de forma normativa e controladora, todavia, para atender às necessidades específicas da população, o seu foco deve estar voltado para a sensibilização do homem e da mulher para a elaboração de um projeto de vida sexual, à luz do Qual, o casal tomará decisão sobre o método contraceptivo, bem como dos planos para conceber filhos ${ }^{(15)}$.

Assim, o sucesso de Programas Educativos em Saúde está em descobrir meios adequados para transmitir informações a determinada população, com a finalidade de levar o público-alvo a obter conhecimentos Que facilitarão, posteriormente, adaptações ou modificações voluntárias de comportamentos Que conduzem à saúde ${ }^{(16)}$

Nesse entendimento, o planejamento familiar precisa utilizar metodologia participativa, com o objetivo de contribuir para que a mulher ou o casal adQuiram conhecimento sobre o próprio corpo e todos os métodos anticoncepcionais. Além disso, deve possibilitar Que os conhecimentos existentes possam ser intercambiados e discutidos, com a finalidade de estimular uma postura crítica e mais esclarecedora sobre esse Programa.

No terceiro eixo - Cuidando do Bebê - composto por duas classes temáticas: classe 3 - Como cuidar do Recém-nascido e Classe 5 o Aleitamento Materno, descreve as representações das gestantes sobre os cuidados com o bebê a partir das suas vivências nas atividades educativas, também entendida como complementar a consulta de enfermagem do pré-natal.

As gestantes evidenciam Que as orientações recebidas nas consultas acerca dos cuidados com o bebê centram-se, prioritariamente, em torno dos cuidados biológicos, sobretudo aqueles que ajudam no controle do processo saúde-doença.

[...] Quando o bebê nascer, ela orientou que é preciso cuidar bem dele, trazer pra fazer a vacina, o teste do pezinho e dar o banho com sabonete neutro, na hora de limpar o umbigo, secar bem e não colocar nada. Observar o corpinho dele, pode ter alergia (Sujeito 4).

É oportuno destacar Que esses cuidados são fundamentais, no primeiro ano de vida do bebê, pois a maioria das causas de óbitos ocorridos nessa faixa de idade seriam preveníveis por imunização, diagnóstico e tratamento precoce de algumas doenças ${ }^{(17)}$.

Além disso, aprende-se que são abordados os principais cuidados com a higiene do bebê, principalmente o banho Que costuma gerar insegurança na mãe devido a fragilidade do bebê. O cuidado com o umbigo também é enfatizado, porQue pode trazer diversas dúvidas, pois existem diversas práticas populares Que causa infecções neonatais graves, até mortes.

É enfatizada a importância do Teste do Pezinho Que diagnostica precocemente a Fenilcetonuria, hipotireoidismo congênito, anemia falciforme e demais hemoglobinopatias. Esta técnica foi introduzida no Brasil na década de 70. Em 1990, a Lei Federal no 8069, de 13 de julho, Que criou o Estatuto da Criança e do Adolescente, obrigou hospitais e demais estabelecimentos de atenção à saúde da gestante a oferecer a triagem neonatal a todas as crianças nascidas ou atendidas em suas instalações. No entanto, somente em 2001, esse Teste tornou-se obrigatório por Lei, Quando o MS institui o Programa Nacional de Triagem Neonatal - PNTN -, em todo o Território Nacional(18).

Para cuidar de um recém-nascido, a mãe precisa aprender práticas apropriadas sobre as necessidades fisiológicas e psicológicas do bebê. Assim, a consulta de enfermagem no pré-natal, constitui-se um espaço importante para ajudá-la na aQuisição dos conhecimentos necessários à realização desses cuidados, além de contribuir para dirimir as dúvidas, os medos e os mitos Que normalmente são suscitados na mãe $e^{(19)}$.

Na classe 5 pode-se observar os conteúdos lexicais relacionados com os cuidados com o aleitamento materno apreendido pelas gestantes. Pode se constatar Que a amamentação é tratada como atributo natural, na Qual o papel social da mulher é o de mãenutriz. As ações educativas baseiam-se na transmissão de informações às mulheres sobre os cuidados para amamentar e as vantagens e desvantagens do aleitamento materno.

Apreende-se que as gestantes representam o leite materno como nutriente Que contribui para o crescimento e desenvolvimento do bebê, conseqüentemente se sentem enaltecidas porQue sabem que são a própria fonte desse alimento.

Fica também evidente nas representações apresentadas pelas gestantes a compreensão de Que o leite materno confere outras vantagens Que beneficiam a mãe, tais como: diminuir o volume do útero, de forma mais rápida; evitar hemorragias no pós-parto, proteger contra a anemia, além de associar-se a um menor risco de câncer de mama.

Além disso, as gestantes explicitaram que o ato de amamentar oportuniza uma maior aproximação entre a mãe e o filho, fortalecendo o vínculo afetivo entre ambos.

Depreende-se Que a lógica das ações propugnadas acerca da amamentação é de informar para responsabilizar, na tentativa de modular o comportamento da mulher em favor da amamentação, imputando-lhe a culpa pelo desmame precoce Que é associada a agravos para a saúde do filho ${ }^{(20)}$.

Destaca-se ainda Que esta forma de abordagem propicia a idealização da prática da amamentação ${ }^{(21,22)}$, Que comumente é acompanhada de sentimentos conflituosos, pois essa prática sofre influência da cultura, das crenças e tabus .

Assim, torna-se necessário Que a enfermeira nesse momento permita à mulher apresentar suas preocupações, temores e expectativas, para poder, a partir desses conhecimentos, desenvolver intervenções Que certamente ajudarão as mulheres a superar as dificuldades Que por ventura possam vir a ocorrer durante o processo da amamentação.

\section{CONSIDERAÇÕES FINAIS}

A gestação é representada como fenômeno complexo e singular, 
Que envolvem diversas complexas mudanças, biológicas, psicológicas, sociais e culturais, demonstrando que os cuidados pré-natais devem ultrapassar a dimensão biológica.

Constatou-se Que a grande maioria das gestantes apresentou representações positivas da consulta de enfermagem do pré-natal, sobretudo devido à forma como se estabelecem as relações de comunicação enfermeira-gestante, em Que são privilegiados o acolhimento e a escuta, superando, assim, em parte, uma prática profissional que tem como eixo central o modelo biológico.

A consulta de enfermagem contribui para Que a gestante enfrente esta etapa da vida com mais traneüilidade, pois lhe permite compreender e expressar os diversos sentimentos vivenciados.
Entretanto, as ações educativas, entendida como atividades contíguas à consulta, Que incluem orientações sobre planejamento familiar e cuidados com o recém-nascido Que abrange a prática e amamentação, pautam-se no modelo tradicional de transmissão das informações, na Qual a mulher é colocada em uma posição passiva, Que impede exploração dos seus conhecimentos prévios, conseqüentemente negociação dos cuidados requeridos.

Faz-se necessário, portanto, para garantir a aderência das gestantes as cuidados trabalhados na consulta de enfermagem, aprofundar a forma de abordagem, principalmente partindo do conhecimento das suas necessidades principais, Que se baseiem no seu modo de vida, na sua cultura.

\section{REFERÊNCIAS}

I. Oliveira JHF. Necessidades assistenciais de saúde da gestante adolescente ao buscar a consulta de enfermagem na unidade básica [dissertação]. Rio de Janeiro (RI) : Escola de Enfermagem Alfredo Pinto, Universidade Federal do Estado do Rio de Janeiro; 2003.

2. Ministério da Saúde (BR). Assistência integral à saúde da mulher: bases de ação programática. Brasília: Centro de Documentação, Ministério da Saúde; 1984.

3. Gama SGN, Szwarcwald CL, Sabroza AR, Branco VC, Leal MC Fatores associados à assistência pré-natal precária em uma amostra de puérperas adolescentes em maternidade do município do Rio de Janeiro (1999-2000). Cad Saúde Pública 2004; 20(supl I): SI OI-SI I I.

4. Costa MC, Guilhem D, Telles MIM. Atendimentos a gestantes no Sistema Único de Saúde. Rev Saúde Pública 2005; 5: 35-9.

5. Ministério da Saúde (BR). Sistema de Informações sobre Nascidos Vivos - SINASC. Brasília; 2004. Disponível em: http:/ /portal.saude.gov.br/portal/svs/visualizar_texto. $\mathrm{cfm}$ ?idtxt $=2 \mathrm{I} 379$

6. Largura M. A assistência do parto no Brasil: uma análise crítica. São Paulo: Gente; 1998.

7. Rios CTF, Vieira NFC. Ações educativas no pré-natal: reflexão sobre a consulta de enfermagem como um espaço para a educação em saúde. Ciênc Saúde Coletiva 2007; 12(2): 477-86.

8. Reinert M. Une méthode d'analyse des données textuelles et une application: Aurelia de G. de Nerval. Bull Meth Sociol 1990; 26: 24-54.

9. Barini R. Aspectos diagnósticos. In: Neme B, editor. Obstetrícia básica. São Paulo: Sarvier; 2000.

10. Szejer M. Uma abordagem psicanalítica da gravidez e do nascimento: saúde perinatal, educação e desenvolvimento do bebê. Brasília: LGE; 2002.

11. Siqueira PRA. A assistência da enfermeira ao parto normal: relatos e atos na visão das mulheres [dissertação]. Rio de Janeiro (RI) : Escola de Enfermagem Anna Nery, Universidade Federal do Rio de Janeiro; 200I

12. BerQuó ES. Planejamento familiar: as faces da falta da informação. Rev Assoc Med Bras 2005; 56: 10-4.

13. Cunha CR. Reflexão bioética na laqueadura tubária em mulheres com desejo de nova gestação [tese]. Brasília (DF) : Faculdade de Ciências da Saúde, Universidade de Brasília; 2005.

14. Bereuó ES, Cavanaghi SM. Fecundidade em declínio: breve nota sobre a redução do número médio de filhos por mulher no Brasil. Novos Estud - CEBRAP 2006; 74: I I-5.

15. Cunha MHCM. Estratégias de sobrevivência entre famílias da perimetral e representações sobre planejamento familiar [dissertação]. Rio de Janeiro (RJ): Escola de Enfermagem Anna Nery, Universidade Federal do Rio de laneiro; 2001.

16. Barbieri M. O enfermeiro na educação de adultos em planejamento familiar [tese]. São Paulo (SP): Escola de Enfermagem, Universidade de São Paulo; 1996.

17. Scochi CGS, Lima RAG, Delácio LB, Morais GR. O conhecimento de puérperas sobre o cuidado com o recémnascido: avaliação da influência da internação em uma maternidade de um hospital-escola. Rev Bras Enferm 1992; 45(I): 36-43.

18. Ministério da Saúde (BR). Programa Nacional de Triagem Neonatal. Brasília: Ministério da Saúde; 2005.

19. Machado MT, Leitão GCM, Holanda FUX. O conceito da ação comunicativa: uma contribuição para a consulta de enfermagem. Rev Latino-am Enferm 2005; 13(5): 23-8.

20. Almeida IAG, Novak FR. Amamentação: um híbrido naturezacultura. I Pediatr 2004; 80(5): I 19-25.

2 I. Silva IA. Amamentar: uma Questão de assumir riscos ou garantir benefícios [tese]. São Paulo (SP): Escola de Enfermagem, Universidade de São Paulo; 1994.

22. Nakano AMS, Mamede MV. A prática do aleitamento materno em um grupo de mulheres brasileiras: movimento de acomodação e resistência. Rev Latino-m Enferm 1999; 7(3): 69-76. 